\title{
Simple beam-optic simulations and proposed mechanical mitigation for the triplet oscillation problem
}

\author{
P. Thieberger, C. Montag, L. Snydstrup, D. Trbojevic, J. E. Tuozzolo
}

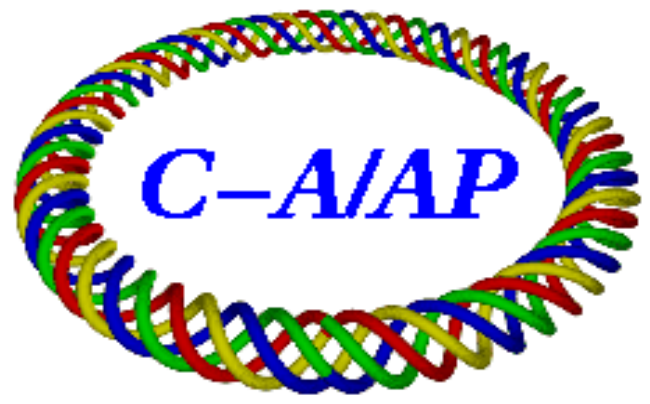

Collider-Accelerator Department Brookhaven National Laboratory Upton, NY 11973

Notice: This document has been authorized by employees of Brookhaven Science Associates, LLC under Contract No. DE-AC02-98CH10886 with the U.S. Department of Energy. The United States Government retains a non-exclusive, paidup, irrevocable, world-wide license to publish or reproduce the published form of this document, or allow others to do so, for United States Government purposes. 


\title{
Simple beam-optic simulations and proposed mechanical mitigation for the triplet oscillation problem
}

\author{
P. Thieberger, C. Montag, L. Snydstrup, D. Trbojevic and J. E. Tuozzolo
}

The purpose of these simulations is to gain a better understanding of the relative contributions to the beam perturbation from the main horizontal oscillation modes (yawing and rolling) of Q1, Q2, and Q3. For this purpose, a simple beam transport program was implemented with an EXCEL spreadsheet to calculate the horizontal beam envelop through the Q1, Q2, Q3 triplet up to the IP, with the possibility of specifying horizontal displacements of the singlets. For now, the weak focusing properties of D0 and DX have been ignored, but could easily be included in the future if necessary. In a first simulation, quadrupole strengths have been adopted that correspond to $\beta^{*}=2 \mathrm{~m}$ at the IP. The parameters used listed in Table 1 were obtained from references [1] and [2].

TABLE 1 Quadrupole triplet parameters

\begin{tabular}{|c||c|c|c|c|c|c||}
\hline $\begin{array}{l}\text { Quadrupole } \\
\text { singlet }\end{array}$ & $\begin{array}{l}\text { Center- } \\
\text { line } \\
\text { distance } \\
\text { from IP }\end{array}$ & $\begin{array}{l}\text { Effective } \\
\text { magnetic } \\
\text { length }\end{array}$ & $\begin{array}{l}\text { Quadrupole } \\
\text { strength for } \\
\beta^{*}=2 \mathrm{~m} \text { at IP }\end{array}$ & Mass & $\begin{array}{l}\text { Physical } \\
\text { length }\end{array}$ & $\begin{array}{l}\text { Diameter } \\
\text { of cold- } \\
\text { mass }\end{array}$ \\
\hline & $(\mathrm{m})$ & $(\mathrm{m})$ & $\left(1 / \mathrm{m}^{2}\right)$ & $(\mathrm{kg})$ & $(\mathrm{m})$ & $(\mathrm{m})$ \\
\hline Q1 & 26.07 & 1.44 & -0.0576 & 1130 & 2.24 & 0.35 \\
\hline Q2 & 30.18 & 3.39 & 0.0561 & 2580 & 4.90 & 0.35 \\
\hline Q3 & 34.80 & 2.10 & -0.0557 & 2260 & 4.37 & 0.35 \\
\hline
\end{tabular}

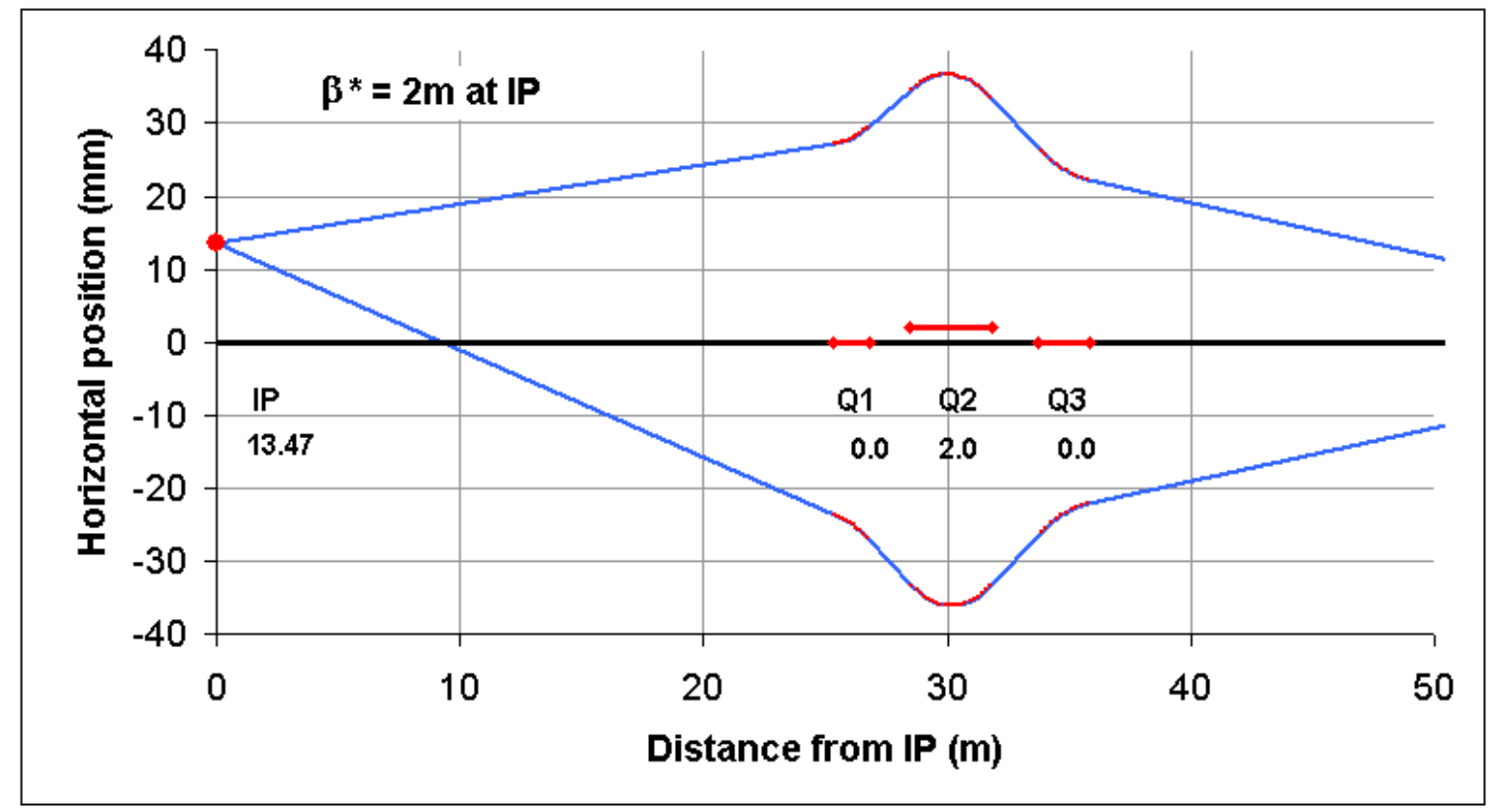

Fig.1 Example where Q2 was displaced horizontally by $2 \mathrm{~mm}$ and the IP moved by $13.47 \mathrm{~mm}$ 
Figure 1 shows an example of the results. In this case Q2 was horizontally displaced by $2 \mathrm{~mm}$, and the IP moved by $13.47 \mathrm{~mm}$. An active Excel chart has been developed where one can use "spin buttons" and "push buttons" to change the lateral displacements of the three elements separately or together (rolling mode), to rotate them (jawing mode), to change the optics from Focusing-Defocusing-Focusing (FDF) to Defocusing-Focusing-Defocusing (DFD), and to select $1 \mathrm{~m}$ or $2 \mathrm{~m}$ values for $\beta^{*}$. Figures 2 and 3 illustrate schematically what "rolling" and "yawing" modes mean, and also indicate measured spring constants [3] appropriate for each motion.

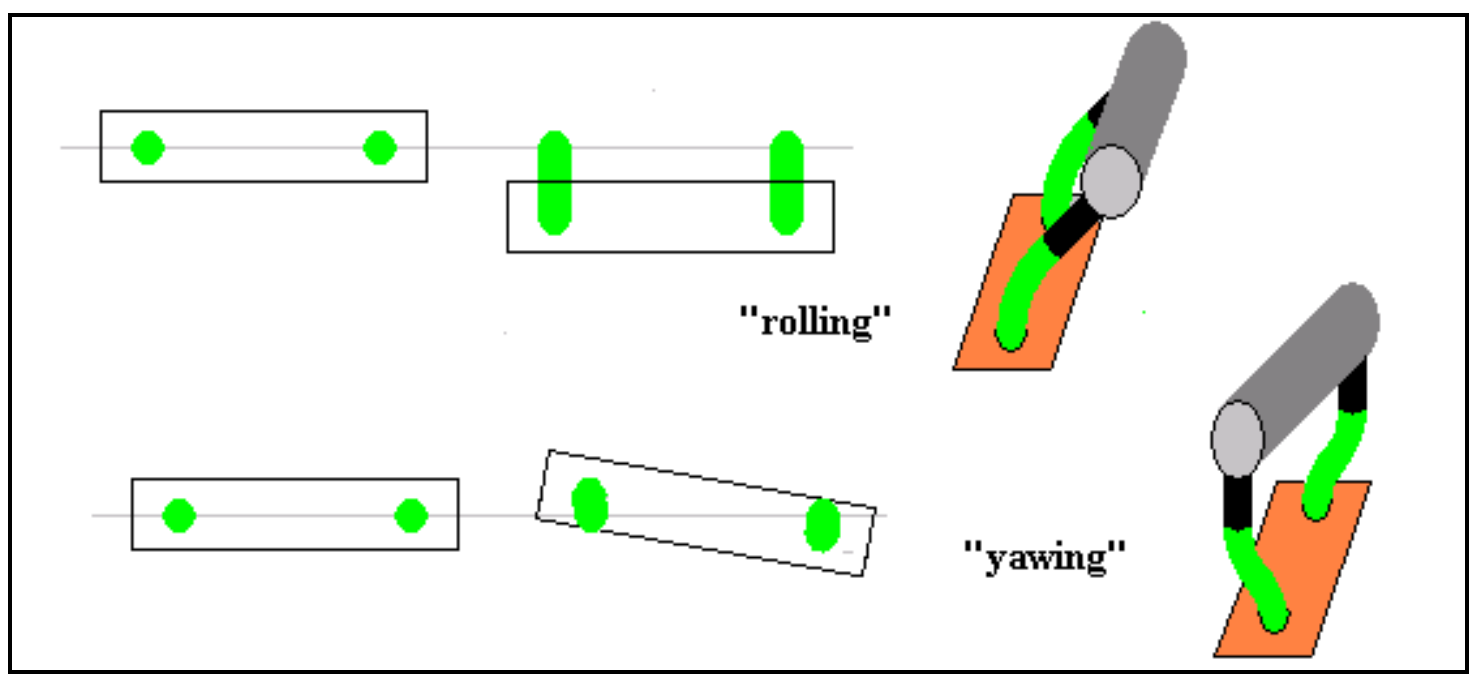

Fig. 2 Schematic representation of the two main oscillation modes.

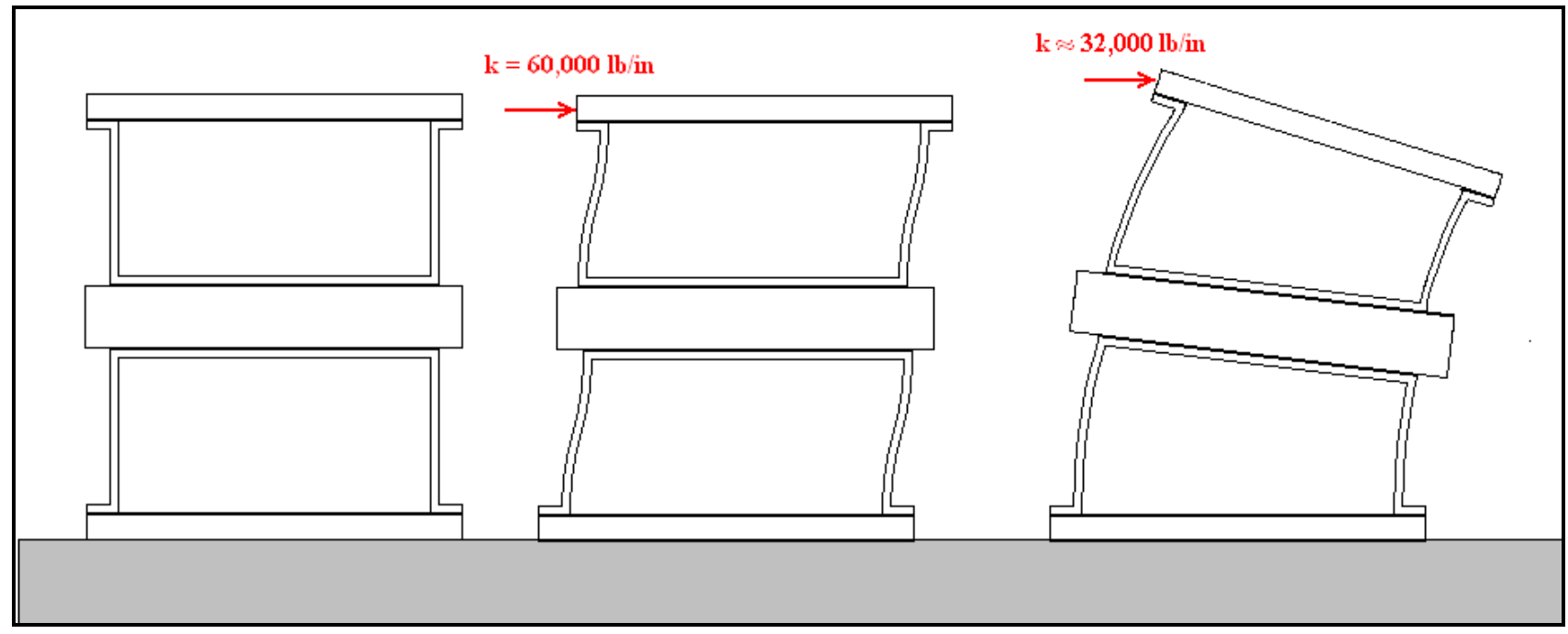

Fig. 3 ULTEM post assemblies shown un-deformed to the left, and deformed in the middle and to the right where the upper surface is respectively constrained or not constrained to remain parallel to the base. Respective measured spring constants are indicated. 
What is actually modeled here is a section of a beam transport, not of a cyclic accelerator. Therefore, the displacement $\Delta \mathrm{X}_{0}$ of the beam at the IP due to a given motion of a quadrupole element will not be the same as the displacement $\Delta \mathrm{X}$ in the ring. But the two beam displacements will be proportional, and the ones from the beam transport can thus be used to obtain relative beam motions values due to different motions of the triplet in the accelerator.

Table 2 shows the $\Delta X_{0}$ values we obtain by displacing the ends of each magnet by $1 \mathrm{~mm}$, either in-phase (parallel displacement or rolling mode) or out-of-phase (rotation around a vertical axis or yawing mode). We also show values for linked displacements for a hypothetical case where the three magnets would be rigidly connected. One of the objectives of these calculations is to determine if it would be advantageous to strengthen the links between the magnets within each triplet.

Table $2 \Delta \mathrm{X}_{0}$ values $(\mathrm{mm})$ for $1 \mathrm{~mm}$ displacements of the ends of the magnets

\begin{tabular}{|c|c|c|c|}
\hline & MODE & $D-F-D$ & $F-D-F$ \\
\hline Q1 & ROLL & -2.20 & 2.12 \\
\hline $\mathrm{Q} 2$ & ROLL & 6.73 & -4.56 \\
\hline Q3 & ROLL & -2.79 & 4.18 \\
\hline Q1 & YAW & 0.04 & 0.00 \\
\hline $\mathrm{Q} 2$ & YAW & 0.02 & 0.01 \\
\hline Q3 & YAW & -0.08 & -0.06 \\
\hline Q1-Q2-Q3 (linked) & ROLL & 1.74 & 1.74 \\
\hline Q1-Q2-Q3 (linked) & YAW & -0.49 & 1.71 \\
\hline
\end{tabular}

The first interesting observation is that yawing motions of individual magnets have a negligible effect upon the beam when compared to the rolling motions. Therefore, if active damping is applied, it will probably be sufficient to damp the motion of the center of each mass, rather than both ends. This should reduce the cost and complication by a factor two. Since in reality there is likely to be coupling between the two main modes, the yawing mode will be attenuated anyway. We also see that the sensitivity to Q2 motion is the largest, with Q3 being a close second in the case of FDF focusing.

We now turn our attention to the possibility of linking the magnets in such a way that, for practical purposes, they move together as a single rigid object. To compare this situation with the present situation where the masses are virtually decoupled, the data of Table 2 is not sufficient. In addition to knowing the beam responses to certain magnet displacements, we must also estimate the relative amplitude of these displacements. This is a more difficult problem since we don't know the origin or nature of the driving forces. As a first approximation we shall assume that these forces can be represented by random horizontal forces of equal rms amplitude applied to both ends of each of the three magnets. We shall further assume that the amplitude of the motion in each case is simply determined by the rms magnitude of the applied force and the corresponding restoring spring constant. This ignores the fact that different mass configurations 
and vibration modes have different resonant frequencies and therefore "sample" different portions of the excitation spectrum. But we can't do much better since we don't know this spectrum.

For the rolling mode the calculation is simple. If we call $\mathrm{F}_{\mathrm{o}}$ the rms amplitude of the horizontal force applied to each end of each singlet, then for one singlet the rms force will be $2^{1 / 2} \mathrm{~F}_{\mathrm{o}}$ while for the linked rigid triplet it would be $6^{1 / 2} \mathrm{~F}_{\mathrm{o}}$. On the other hand the restoring spring constant is 3 times larger in the linked case. Therefore, with our approximations, the estimated amplitude for the linked case in the rolling mode will be $3 * 2^{1 / 2} / 6^{1 / 2}=3^{1 / 2}=1.73$ times smaller. For estimating the amplitude of the linked case in the yawing mode, we follow the same procedure, but now constraining the center-of-mass point to be fixed and taking into account the springconstant which is larger by a factor $\sim 1.9$ in this case (see Fig. 3). Compared to the linked rolling mode, the amplitude of the motions for the linked yawing mode is 1.69 times smaller. These results are utilized in Table 3 to calculate the effects on the beam position ( $\mathrm{X}_{0}$ values), as was done in Table 2, but now with estimated relative vibration amplitudes rather than the $1 \mathrm{~mm}$ displacements assumed before.

Table 3 Relative $\Delta \mathrm{X}_{0}$ values for the estimated relative magnet vibration amplitudes (see text)

\begin{tabular}{|c|c|c|c|}
\hline & MODE & $\mathrm{D}-\mathrm{F}-\mathrm{D}$ & $F-D-F$ \\
\hline$\overline{\mathrm{QQ1}}$ & ROLL & -1.56 & 1.50 \\
\hline Q2 & ROLL & 4.76 & -3.22 \\
\hline Q3 & ROLL & -1.97 & 2.95 \\
\hline Q1 & YAW & 0.02 & 0.00 \\
\hline $\mathrm{Q} 2$ & YAW & 0.01 & 0.04 \\
\hline Q3 & YAW & -0.03 & -0.02 \\
\hline Addition in quadrature & & 5.39 & 4.62 \\
\hline "Q1-Q2-Q3 (linked) & ROLL & 0.71 & 0.71 \\
\hline Q1-Q2-Q3 (linked) & YAW & -0.12 & 0.41 \\
\hline Addition in quadrature & & 0.719 & 0.821 \\
\hline
\end{tabular}

To estimate the overall effect of the vibrations upon the beam we also calculate in Table 3 the additions in quadrature of the effects of the two linked vibrations, and separately of the effects of the six not linked motions. We see that this estimate leads to an average factor of $\sim 6.5$ reduction in beam fluctuation amplitude by rigidly linking the three singlets within each triplet. This factor was initially expected to be larger of $\beta^{*}$ values at the IP smaller than the $2 \mathrm{~m}$ assumed here. However, the same calculations were repeated for $\beta^{*}=1 \mathrm{~m}$, and an estimated reduction of $\sim 6.3$ was obtained, i.e. not significantly different than for $2 \mathrm{~m}$. The explanation is that the focusing for different $\beta^{*}$ values is really accomplished mostly by the area Q9-Q10 quadrupoles, and that the gradients in the triplets do not differ to much between $\beta^{*}=1 \mathrm{~m}$ and $\beta^{*}=2 \mathrm{~m}$. 
There should in principle be no problems associated with the transverse rigidizing of the connections between singlets within each triplet [4] since they move together during cool down. One possible implementation would consist in replacing the presently installed tie-rods by larger diameter, perhaps hollow, cylinders [5]. Another possibility would be to consider split cylindrical "clam shells" that fit over the cold masses of adjacent magnets.

Such mechanical improvements would not cause increased cooling requirements. Should a factor of $~ 6.5$ amplitude reduction be insufficient, any further active or passive measures would be simplified and made more economical by requiring a smaller improvement and fewer cold-mass attachment points. It seems therefore that rigid connections between Q1, Q2 and Q3 should be the first step.

\section{Possible Implementations}

We now turn to the question of what type of mechanical connection between the singlets could be implemented to achieve to a large extent the advantages outlined in the previous section. The present arrangement is shown in Fig. 4. In this top view, one sees only the top 1" diameter tie rods used between consecutive singlets. The bottom tie rods are diametrically opposed to the top ones. The ends of these tie rods are threaded and attached to large lugs welded to the cold masses by means of nuts and spherical washers to accommodate slight misalignments. Even if these attachments are considered rigid, the 1" diameter tie rods are not substantial enough to affect the vibrations very substantially. This was shown in detailed ANSYS simulations [6], and, as seen below, we will arrive at the same conclusion with our simplified models.

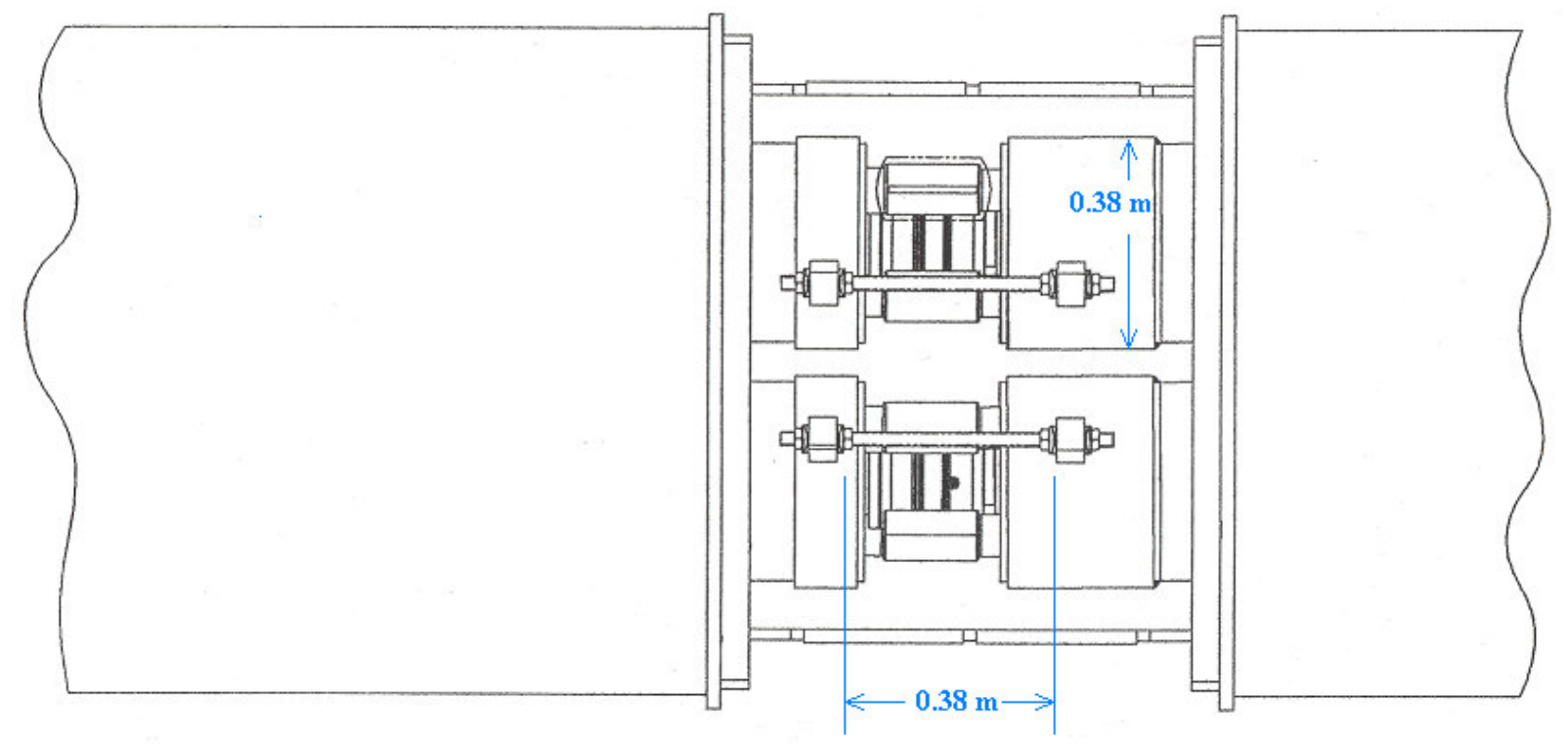

Fig. 4 Present tie rod connections between $Q 1$ and $Q 2$, and between $Q 2$ and $Q 3$. In this top view only the top tie rods are visible. Identical tie rods are installed at the bottom; diametrically opposed to the top ones. The tie rods are 1" diameter stainless steel with treaded ends. They are fastened to lugs welded to the cold masses by means of nuts and spherical washers to accommodate small misalignments. Indicated in blue numbers are two dimensions not given in Table 1; namely the spacing between lugs and the diameter of the end volumes. 
The two solutions contemplated here are illustrated schematically in Fig. 5. On the one side we show additional tie rods. And on the other side we suggest the possibility of connecting the two magnets with a cylindrical stainless steel shell or sleeve welded, or otherwise fastened to the singlets at each end. We will try to estimate what would be required to achieve close-to-rigid motion of the joint triplet masses. In the case of the tie rods we will vary their number and diameter, and in the case of the sleeve we will vary its thickness

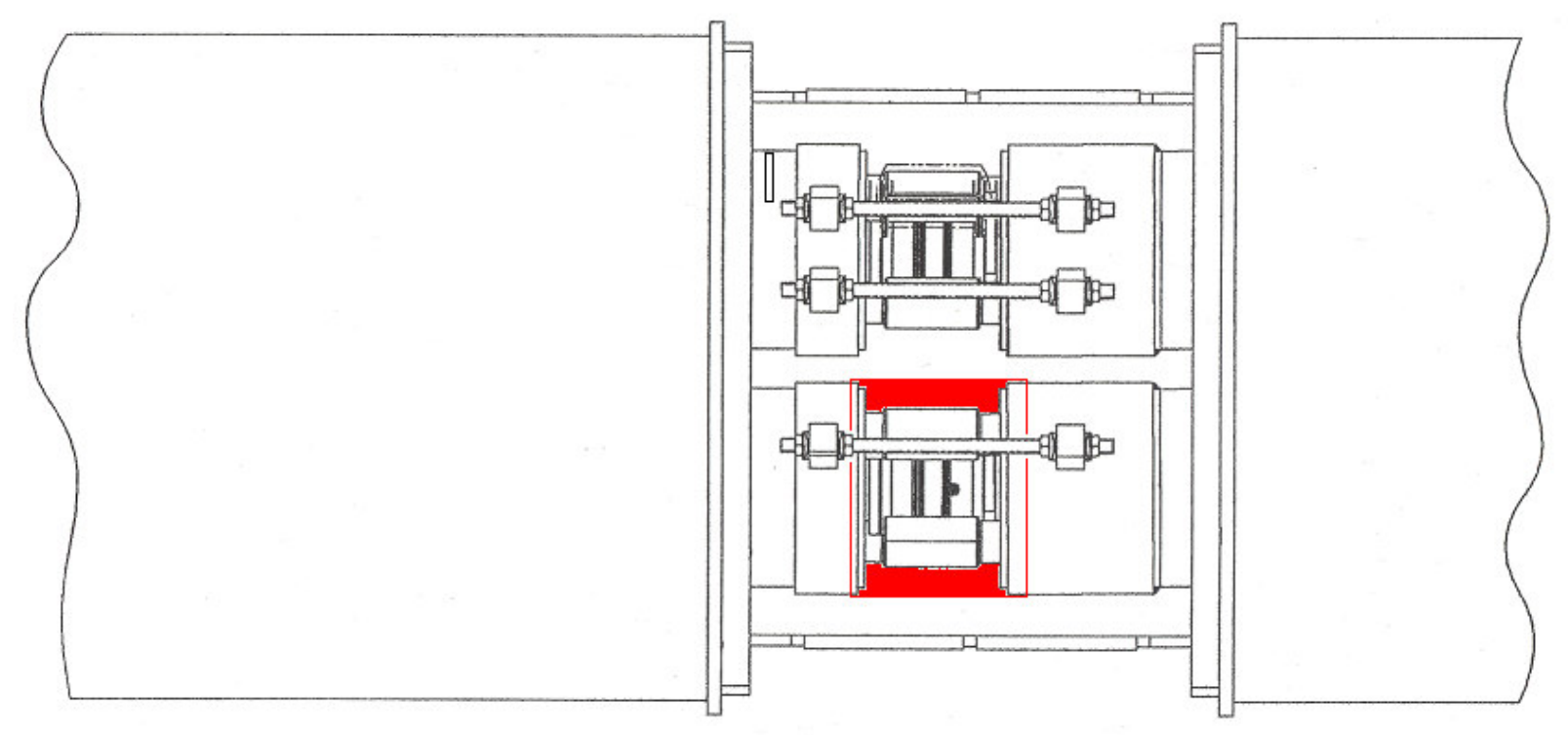

Fig. 5 Schematic illustration of the two solutions analyzed in the present work to increase the rigidity of the Q1-Q2 and Q2-Q3 connections in order to ensure that the three singlets move as nearly as possible together as a rigid body. At the top it is shown how the number of tie bars may be increased, and we investigate a range of possible numbers and diameters. At the bottom it is suggested that a cylindrical stainless steel shell or sleeve may be fastened to the cold masses, and we obtain results as function of the wall thickness of this shell.

While complete detailed simulations may be desirable and will perhaps be performed before a design is implemented, the purpose here is to roughly estimate in a simple way the manner in which the design parameters of the additional supports will affect the vibrations. For that purpose we study the two types of motions considered above (yawing and rolling, see Fig. 2), but now disregarding the main magnet supports, and taking only into account the forces and torques generated when the interconnecting structure (tie rods or sleeve) is deformed. We will have achieved our goal of nearly rigid motion if the corresponding eigen-frequencies are considerably higher than the $\sim 10 \mathrm{~Hz}$ characteristic of the spring constants of the main supports.

Top views of the motion configurations used for the calculations are shown in Fig. 6. Only Q2 and Q3 were modeled, as they have the largest and similar masses and lengths (see Table 1). Intermediate values were adopted for these estimates, thus making the magnet pair symmetric to simplify the calculations. In Fig 6a we illustrate the yawing or rotational motion of the two 
coupled masses while $6 \mathrm{~b}$ shows the rolling or translational motion. In this last case the masses are either assumed to be guided to remain parallel to each other, or equivalently (as shown in Fig. 6c) a spring with the appropriate spring constant is imagined to be attached at the center-ofmass position. For strong tie rod or shell coupling this mode is fictitious and will in reality not have a significant amplitude. This is precisely the purpose of this exercise; to find a strong enough connection (high enough eigen frequency) to ensure that this type of motion be largely suppressed.

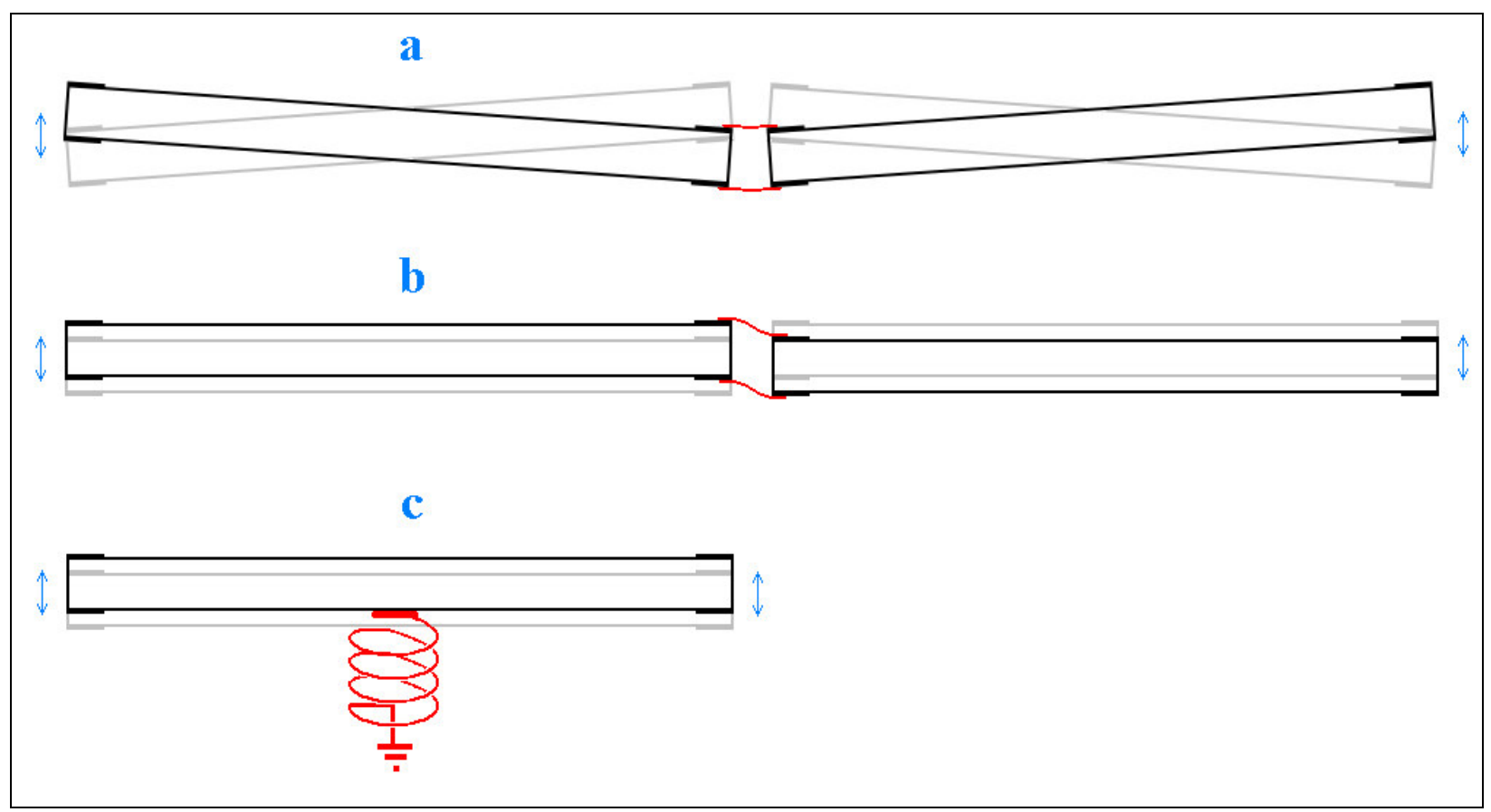

Fig. 6 Top views of the simplified motion configurations used for the calculations. The only forces that are considered are those due to the deformations of the tie rods or of the cylindrical shell. Only Q2 and Q3 were modeled, as they have the largest and similar masses and lengths (see Table 1). Intermediate values were adopted for the estimates making the magnet pair symmetric to simplify the calculations. a) Yawing motion of the two coupled masses (see Fig. 2). b) Rolling motion of the two coupled masses. Here the masses are either assumed to be guided to remain parallel to each other, or equivalently c) a spring with the appropriate spring constant is imagined to be attached at the center-of-mass position.

The frequencies for translation and for rotation $\left(f_{t}\right.$ and $f_{r}$ respectively) are calculated using the harmonic oscillator equations:

$$
f_{t}=\frac{1}{2 \pi} \sqrt{\frac{k_{t}}{M}}
$$


$f_{r}=\frac{1}{2 \pi} \sqrt{\frac{k_{r}}{I_{M}}}$

Where $\boldsymbol{M}$ is the mass, $\boldsymbol{I}_{\boldsymbol{M}}$ the moment of inertia, $k_{t}[\mathrm{~N} / \mathrm{m}]$ is the translational spring constant and $k_{r}[\mathrm{Nm} / \mathrm{radian}]$ is the rotational spring constant.

Table 4 lists the values of the quantities used to calculate these frequencies. The other quantities that determine the frequencies are the number $\boldsymbol{N}$ of tie rods and their diameter $\boldsymbol{D}$, and the thickness $\boldsymbol{T}_{s}$ of the shell.

Table 4. Some of the quantities used to calculate the frequencies

\begin{tabular}{|l|c|r|c|}
\hline Quantity & Symbol & Value & Unit \\
\hline Mass (average of Q1 and Q2) & $\boldsymbol{M}$ & 2420 & $\mathrm{~kg}$ \\
\hline Average length & $\boldsymbol{L}$ & 4.64 & $\mathrm{~m}$ \\
\hline Average moment of inertia & $\boldsymbol{I}_{\boldsymbol{M}}$ & 4400 & $\mathrm{~kg} \mathrm{~m}^{2}$ \\
\hline Stainless steel modulus of elasticity & $\boldsymbol{E}$ & 193,000 & $\mathrm{MPa}$ \\
\hline Inner radius for supports (rods or shell) & $\boldsymbol{R} \boldsymbol{s}$ & 0.21 & $\mathrm{~m}$ \\
\hline Distance between support attachment points & $\boldsymbol{l}$ & 0.38 & $\mathrm{~m}$ \\
\hline
\end{tabular}
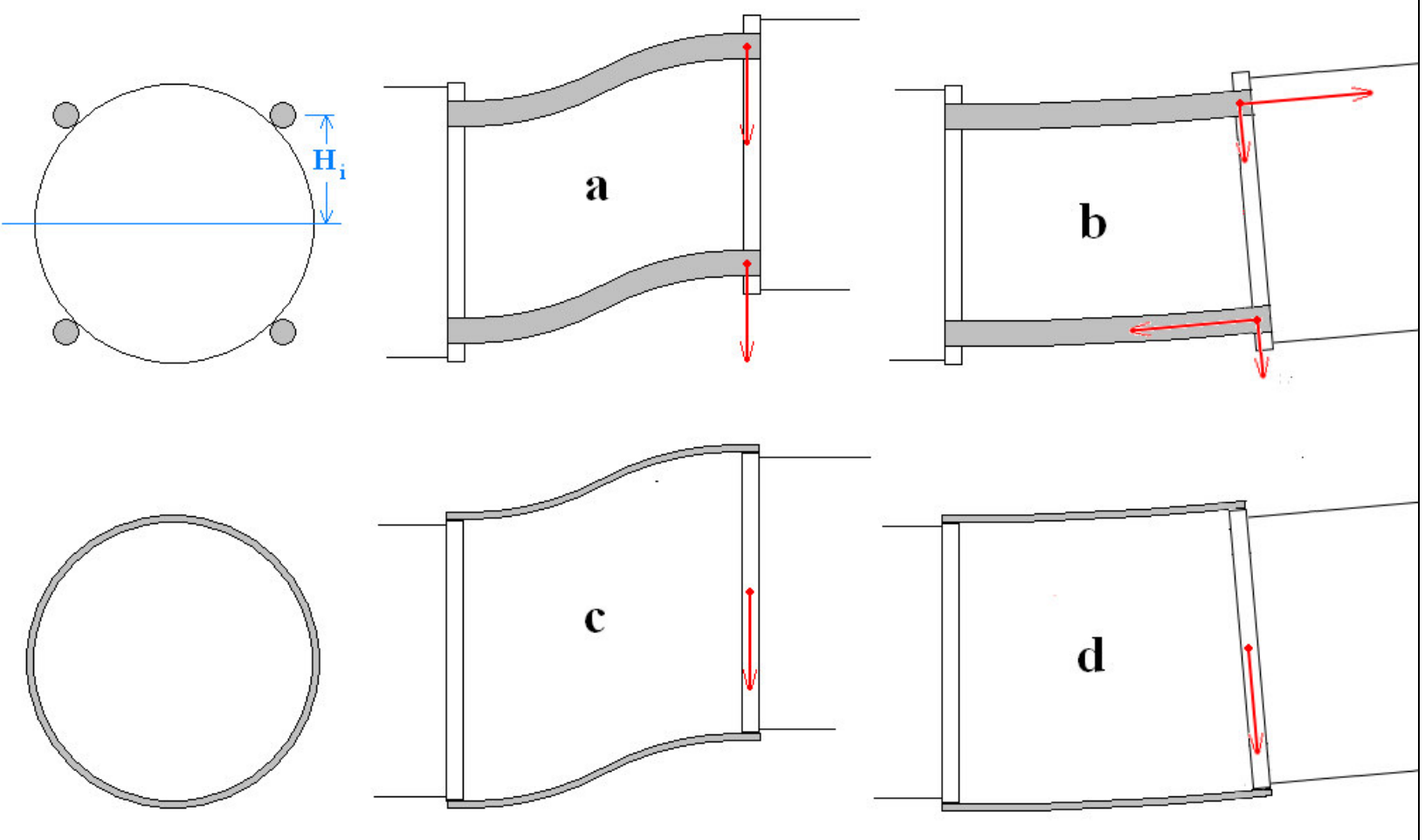

Fig. 7 Schematic of the tie rods and shell deformations for the two type of motion depicted in Fig. 6. Examples for 4 tie rods are shown in the upper part of the figure ( $a$ and $b$ ), while the lower part ( $c$ and $d$ ) show the deformations of a cylindrical shell. 
The tie rods and shell deformations for the two type of motion depicted in Fig. 6 are shown in Fig. 7. To calculate the spring constants, we calculate the forces acting on the masses to the right caused by these deformations while the masses to the left are assumed to be stationary for this purpose. In reality both masses move in equal and opposite directions for the symmetric case considered here. Therefore the effective spring constants for calculating the frequencies will be twice the values obtained here. Examples for 4 tie rods are shown in the upper part of the figure ( $a$ and $b$ ), while the lower part ( $c$ and d) show the deformations of a cylindrical shell. Standard deflection formulas for beams [7] can be used for cases a, c and d.

For case $b$, the most important contributions arise from the stretching of the tie bars on one side and their compression on the other. For the range of tie bar diameters considered here, their bending in case $\mathrm{b}$ makes an almost negligible contribution to the spring constant, but this contribution was nevertheless included in the calculations.

The deflection formulas for beams were obtained as special cases of more general formulas given e.g. in reference 7. For one end fixed and the other end guided so as to prevent rotation (cases a and $\mathbf{c}$ of Fig. 7), the displacement $\boldsymbol{y}$ for a force $\boldsymbol{F}$ applied at the moving end is:

$$
y=\frac{F \times l^{3}}{12 \times E \times I}
$$

where $\boldsymbol{I}$ is the area moment of inertia about the centroidal axis of the beam cross section, which for circular cross sections is given by:

$$
I=\frac{\pi}{4}\left(r^{4}-r_{i}^{4}\right)
$$

where $\boldsymbol{r}$ is the outer radius of the beam and, $\boldsymbol{r}_{\boldsymbol{i}}$ is the inner radius in the case of an annular cross section (as is the case for the shell).

For one end fixed and the other end free (case $\mathbf{d}$ of Fig. 7), the displacement $\boldsymbol{y}$ and the deflection angle $\Theta$ for a force $\boldsymbol{F}$ applied at the moving end are:

$$
\begin{aligned}
& y=\frac{F \times l^{3}}{3 \times E \times I} \\
& \theta=\frac{F \times l^{2}}{2 \times E \times I}
\end{aligned}
$$


The spring constants $\boldsymbol{k}_{\boldsymbol{t}}$ for calculating the translational frequencies (cases of Figs.7a and 7c) are then simply $2 \times \boldsymbol{F} / \boldsymbol{y}$ obtained from 5):

$k_{t}=N \times \frac{24 \times E \times I}{l^{3}}$

where $\boldsymbol{I}$ is given by 6$)$ either for each of the $\boldsymbol{N}$ solid tie rods $\left(\boldsymbol{r}_{\boldsymbol{i}}=\boldsymbol{O}\right.$ in this case) or for the cylindrical shell ( $N=1$ in this case).

The rotational spring constant for the case of Fig $7 \mathrm{~d}$ is twice the ratio of the torque or moment of the force $\boldsymbol{F}$ given by 8 ) with respect to the center of mass of the magnet divided by the deflection angle $\boldsymbol{\Theta}$.

$$
k_{r}=\frac{2 \times 2 \times E \times I \times L / 2}{l^{2}}=\frac{2 \times E \times I \times L}{l^{2}}
$$

And finally, for the case of Fig. 7b, there is a contribution to the rotational spring constant from the bending of each of the $N$ tie-rods given approximately by equation 10) with the appropriate value of $\boldsymbol{I}$. But by far the largest contribution comes from the moments with respect to the center of mass of the longitudinal forces generated by stretching and compressing the individual tie rods. If we call $\boldsymbol{H}_{\boldsymbol{i}}$ the distance of tie rod \#i from the median plane (see Fig 7) then its contribution due to stretching or compressing to the rotational spring constant will be:

$$
k_{r, i}=\frac{2 \times E \times\left(\pi \times D^{2} / 4\right) \times H_{i}^{2}}{l}=\frac{\pi \times E \times D^{2} \times H_{i}^{2}}{2 \times l}
$$

\section{RESULTS}

An Excel spreadsheet was prepared to carry out the eigen frequency calculations described above. Some results obtained with this spreadsheet are shown in Fgs 7 and 8

Figure 7 shows results of the resonant frequencies of the systems sketched in Fig. 6 for the case of six stainless steel tie rods (uniformly spaced) as a function of their diameter. This is an overly optimistic example, since there is probably not enough space to accommodate this number of tie rods, especially not for the larger diameters. We see that for the "translation" or rolling mode (which is the one of concern), one barely reaches an eigen frequency of $\sim 10 \mathrm{~Hz}$ even if one could install six 1" diameter tie rods. Thus, increasing the number of tie rods and/or their diameter doesn't seem to be a viable solution. 


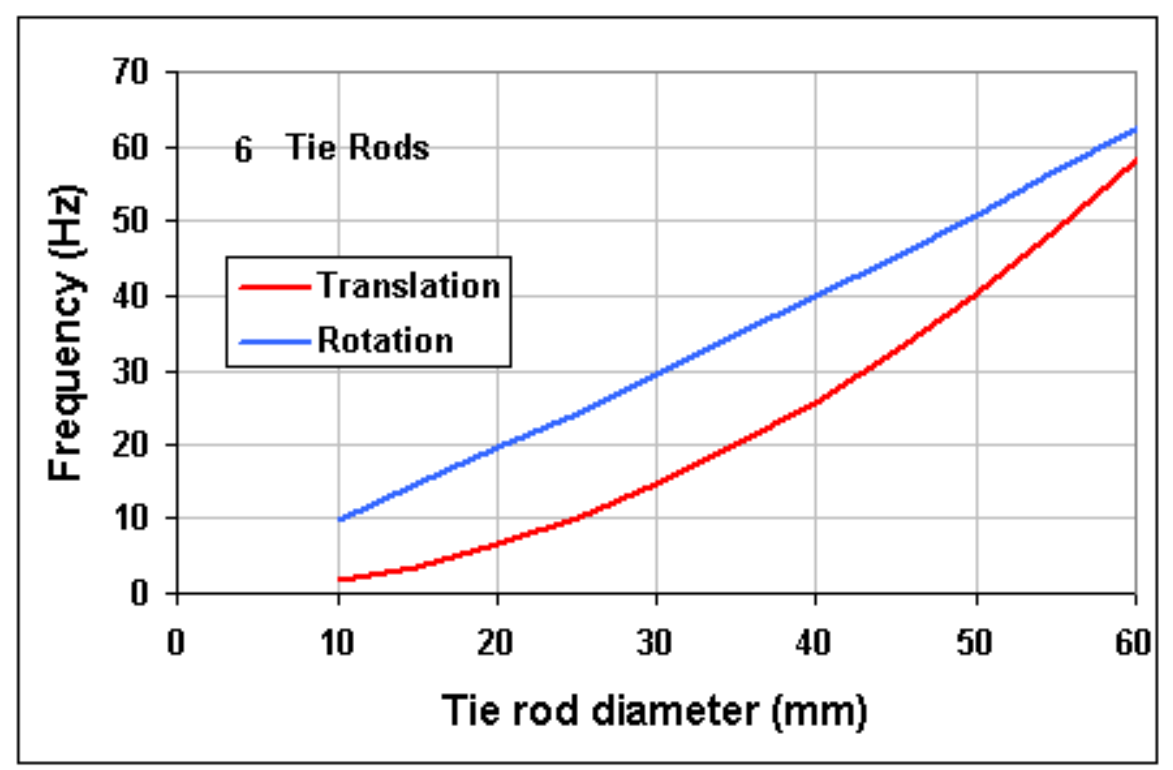

Fig. 7 Results of the resonant frequencies of the systems sketched in Fig. 6 for the case of six stainless steel tie rods (uniformly spaced) as a function of their diameter. This is an overly optimistic example, since there is probably not enough room to accommodate this number of tie rods, especially not for the larger diameters. We see that for the "translation" or rolling mode (which is the one of concern), one barely reaches an eigen frequency of $10 \mathrm{~Hz}$ even if one could install six 1" diameter tie rods. Ths, increasing the number of tie rods and/or their diameter doesn't seem to be a viable solution.

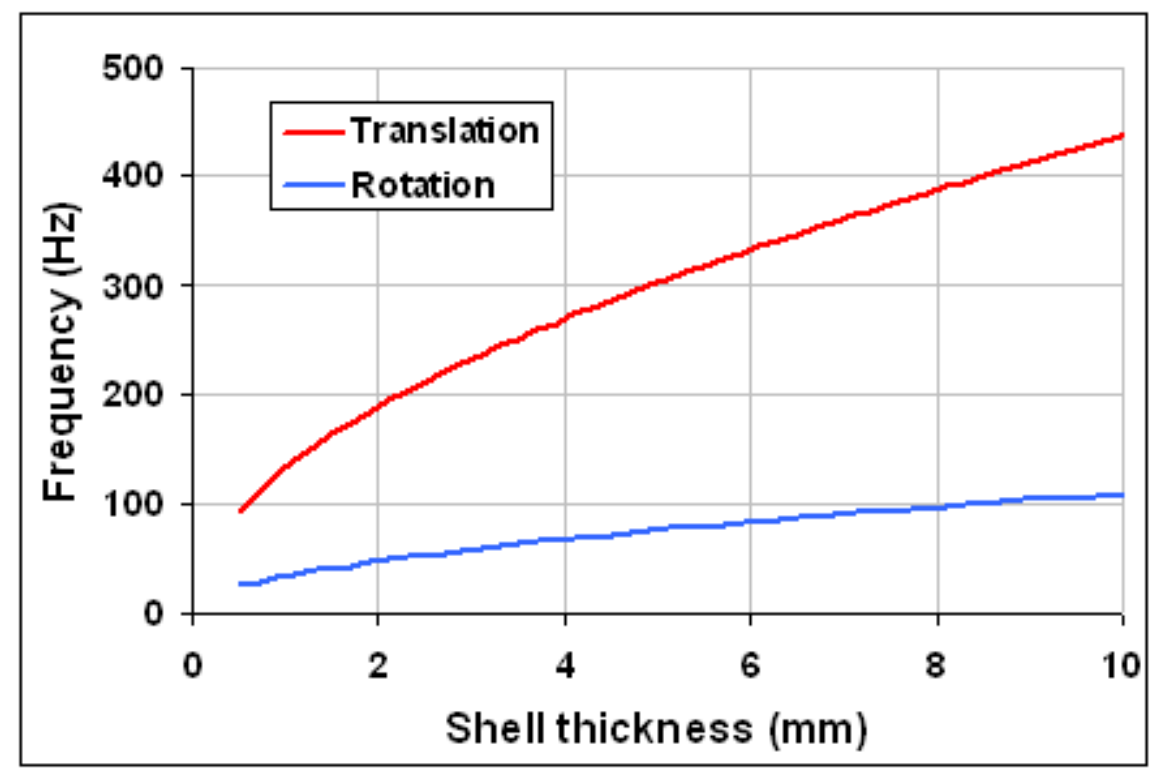

Fig. 8 Results of the resonant frequencies of the systems sketched in Fig. 6 for the case of a cylindrical stainless steel sleeve or shell welded to the cold masses as indicated in the lower part of Fig. 5. The eigen frequencies are plotted as function of the shell thickness. We see that for thicknesses larger than 2 or $3 \mathrm{~mm}$, frequencies well above $10 \mathrm{~Hz}$ are obtained, especially for the motion that is of greatest concern for its relatively large effect on the beam. 
In contrast, the results shown in Fig 8 are much more promising. We see here the estimated resonant frequencies of the systems sketched in Fig. 6 for the case of a cylindrical stainless steel sleeve or shell welded to the cold masses as indicated in the lower part of Fig. 5. The eigen frequencies are plotted as function of the shell thickness. We see that for thicknesses larger than say 2 or $3 \mathrm{~mm}$, frequencies well above $10 \mathrm{~Hz}$ are obtained, especially for the translation-type motion which is of greatest concern for its relatively large effect on the beam.

\section{CONCLUSIONS}

A relatively thin cylindrical stainless steel shell, perhaps $1 / 8$ " thick, welded or otherwise rigidly attached to the ends of the cold masses would achieve to a large extent the factor $\sim 6$ reduction in $10 \mathrm{~Hz}$ beam motions estimated in the first part of this report. Such a shell could probably be fabricated in two halves, perhaps with overlapping longitudinal seams fastened rigidly together with screws or welds in order to preserve the full strength of the cylindrical shell.

There may be technical difficulties in implementing this change, such as fitting the shells to the non-concentric "end volumes" or due to welding-induced misalignments. These would need to be considered carefully in the design modification. The positions of the magnets must not be affected by more than 1 or 2 mils, which is the present surveying tolerance. Surveys would be performed before and after the installation to ensure that no excessive displacements have occurred.

One could consider providing easier access to the enclosed volume by making part of this shell a removable panel fastened with screws. The effective strength of the connection would not be significantly affected as long as good metal-to-metal contact is ensured along the edges of this panel. Since elastic forces are small for micron-size vibrations, frictional forces will be sufficient to totally prevent any slippage.

\section{REFERENCES}

1) C. Montag, M. Brennan, J. Butler, R. Bonati, P. Koello, "Observation of Mechanical Triplet Vibrations in RHIC", Proc. 26th Advanced ICFA Beam Dynamics Workshop on Nanometer Size Colliding Beams (NANOBEAM) 2002, CERN-Proceedings2003-001

2) Dejan Trbojevic, private communication.

3) John Sondericker, private communication.

4) John Sondericker, private communication.

5) Joseph Tuozzolo, private communication.

6) ANSYS simulations by J. Cozzolino

7) Formulas for Stress and Strain by R. J. Roark and W. C. Young - fifth edition McGraw - Hill 1988. 